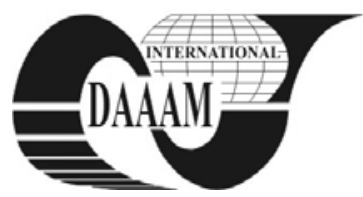

\title{
RESEARCH ON THE POWER LOSS IN THE HYDROSTATIC VALVES
}

\author{
TOPLICEANU, L[iliana]; BIBIRE, L[uminita] \& GHENADI, A[drian] S[telian]
}

\begin{abstract}
The hydrostatic drive systems can be used in multiple fields, from machine tools to industrial robots and sophisticated hydronic systems. Its wide range of use is due to the possibility of developing great force and momentum, which is difficult to achieve with other types of actuators. One of the disadvantages of these systems is the power loss through the circuit elements used to adjust the speed of the hydrostatic engines. The paper presents the results of research performed on a throttle using different pressure values and it establishes the cases in which the use of the throttle is efficient.
\end{abstract}

Key words: hydrostatic, throttle, loss of pressure

\section{INTRODUCTION}

Within any mechanical system, the most important functional parameters, the ones that actually impose its designing are:

- $\quad$ the momentum $\mathrm{M}_{\mathrm{T}}$ quantified in $\mathrm{Nm}$ and

- the rotational speed $n$ quantified in rotations/min.

The following specific measurements for the hydraulic actuation systems correspond to these mechanical parameters:

- $\quad$ pressure $p$ in bars for the momentum $M_{T}$ and

- $\quad$ flow $\mathrm{Q}$ quantified in $\mathrm{l} / \mathrm{min}$ corresponding to the speed and the rotational speed $\mathrm{n}$ respectively.

The difficult adjustment and the inter-relation between these measurements are important problems that influence and restrict the use of the hydrostatic actuator systems.

\subsection{Adjusting the speed of the hydraulic motors}

Adjusting the speed of the rotary or linear hydrostatic motors is achieved through two basic methods:

- the volume method - which consists in using the pumps with adjustable flow; this method is recommended in the case of high power circuits, of car start-up, etc.;

- the resistive method - which consists in placing some fixed and variable resistors on the motor circuit; this method ensures a fine adjustment, on narrow fields of pressure.

Both methods have important disadvantages, well known in the literature, which we are not going to insist on right now. The complex driven systems often include both types of adjustments (Oroveanu, 1967; Topliceanu, 2003).

\section{CIRCUIT ELEMENTS FOR THE RESISTIVE ADJUSTMENT}

As a general theoretical principle, the adjustment of speed in the case of hydrostatic motors is achieved by adjusting the quantity of liquid which operate them. In order to achieve this, the resistive method uses circuit elements - the so-called throttles - which control the flow section on inflow, outflow or, in the case of paralleling assemblage, it sends a certain quantity of liquid directly to the tank. The throttles come in extremely varied shapes, adapted to the different operational conditions and to the different viscosity of liquids. An extremely important phenomenon which takes place while using these resistors is the heating of the liquid, which leads to a modification of the viscosity and implicitly of the flow that goes through the throttle. From this point of view, the throttles with serial diaphragms (Fig. 1), have a very good behaviour (Tita, 2009).

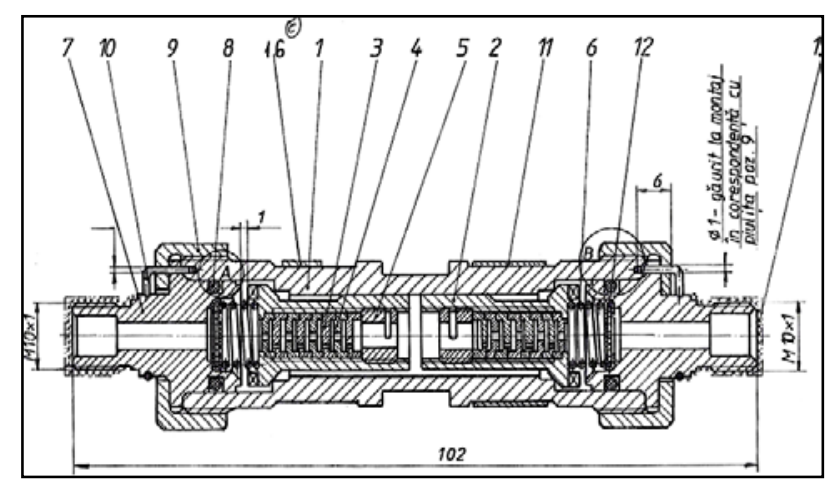

Fig. 1. Valve throttle SD 3590 1- Body; 2-Valve; 3,4Diaphragm; 5- Threaded ring; 6- Spring; 7- Connector; 8Sealing system; 9 - Nut; 10 - Ring; 11 - Label; 12 - Filter

\subsection{Deter mining the pressure fall on the thr ottle}

Inserting the throttles on the motor circuit leads to a pressure fall on each throttle. The calculation of the pressure loss by means of the hydraulic resistance is achieved based on the pressure loss coefficient. The value of this coefficient depends on the characteristics of the flow, so on the Reynolds number.

The pressure loss is determined according to the following equations (***MathWorks):

$$
\Delta p=\left\{\begin{array}{c}
K \cdot \frac{\rho}{2 A^{2}} q|q| \text { for } R e>\operatorname{Re}_{c r} \\
K \cdot \operatorname{Re} e_{c r} \frac{v \cdot \rho}{2 D_{H} \cdot A} q \text { for } R e \leq \operatorname{Re}_{c r}
\end{array}\right.
$$

In this relation

$$
\Delta p=p_{1}-p_{2}
$$
is the difference between the inlet and the outlet pressure on the throttle level, $\mathrm{K}$ is the pressure loss coefficient of the hydraulic resistor $\quad K=\left\{\begin{array}{l}\text { const } \\ K(R e)\end{array}\right.$

and for the Reynolds number and the flow section through the port we use the formulas: $R e=\frac{q D_{H}}{A v}, \quad D_{H}=\sqrt{\frac{4 A}{\pi}}$.

The other notations are:

$q$ - flow rate;

A - passage area;

$\mathrm{D}_{\mathrm{H}}-$ oriffice hydraulic diameter;

$\rho$ - fluid density;

$v$ - fluid kinematic viscosity.

In many theoretical studies (Vasiliu, 1993) it is considered that the flow passing through a throttle varies linearly with the section of the passage hole/area. However, in practice it must be corrected by a certain value due to the viscous friction of the agent inside the motor during the flow, as it results from the formulas 1 . 


\section{EXPERIMENTAL RESEARCH}

The experimental research was performed in cooperation with the Reliability Laboratory at S.C.Aerostar S.A. Bacau and it consisted in making a throttle (Fig. 1), determining the pressure loss for different values of the inlet pressure, and making automatic diagrams using the CATMAN programme.

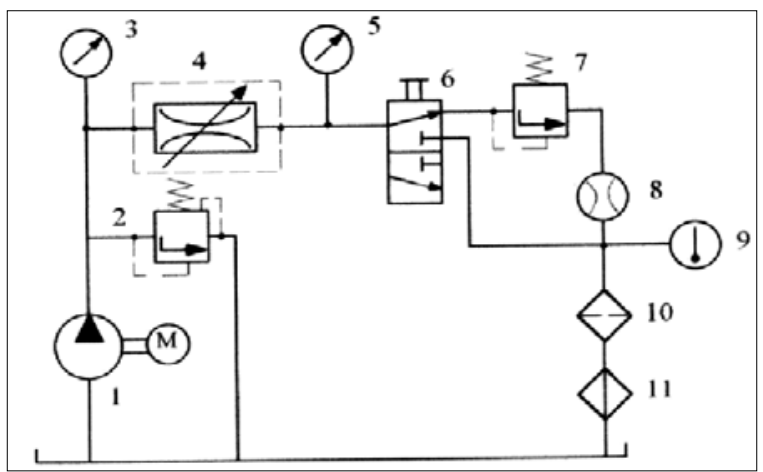

Fig. 2. Scheme of the stand: 1- pump; 2- safety valve; 3 and 5 -manometer; 4 -throttle; 6 -distributor; 7 - valve; 8 debimeter; 9 -thermometer; 10 -filter; 11 -heat shift

In order to establish the inlet and the outlet pressure, two pressure transducers, working in the area 0-400 bar were assembled (Fig. 3). These gauges were connected to a MGC (Hottinger) measurement device as an interface with the PC unit which has a Catman measurement programmed installed which automatically draws diagrams according to the readings of the gauges. The MGC device can measure simultaneously 16 parameters (non-electrical) and can simultaneously draw evolution diagrams for the parameters.

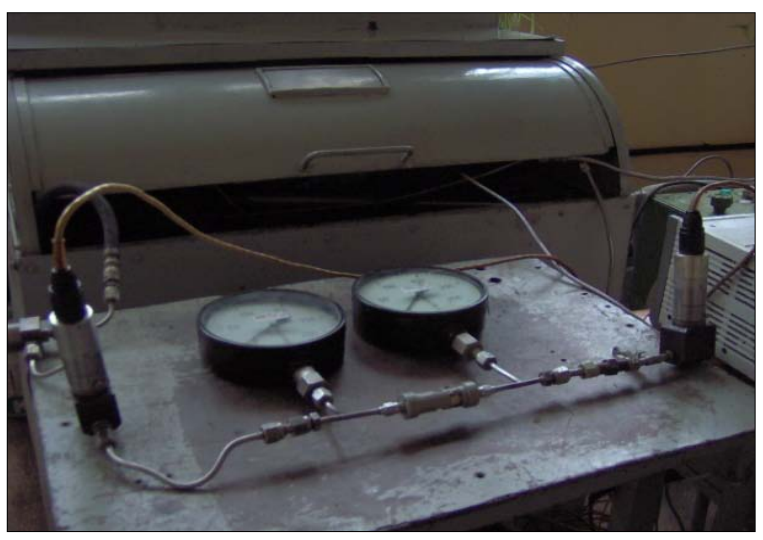

Fig. 3. Throttle test stand

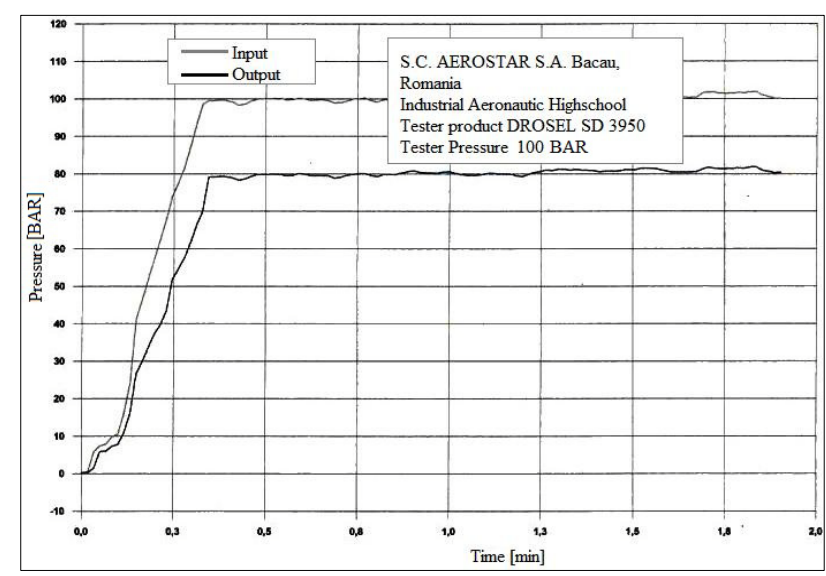

Fig. 5. Pressure drop on the throttle for $\mathrm{p}_{1}=100 \mathrm{bar}$

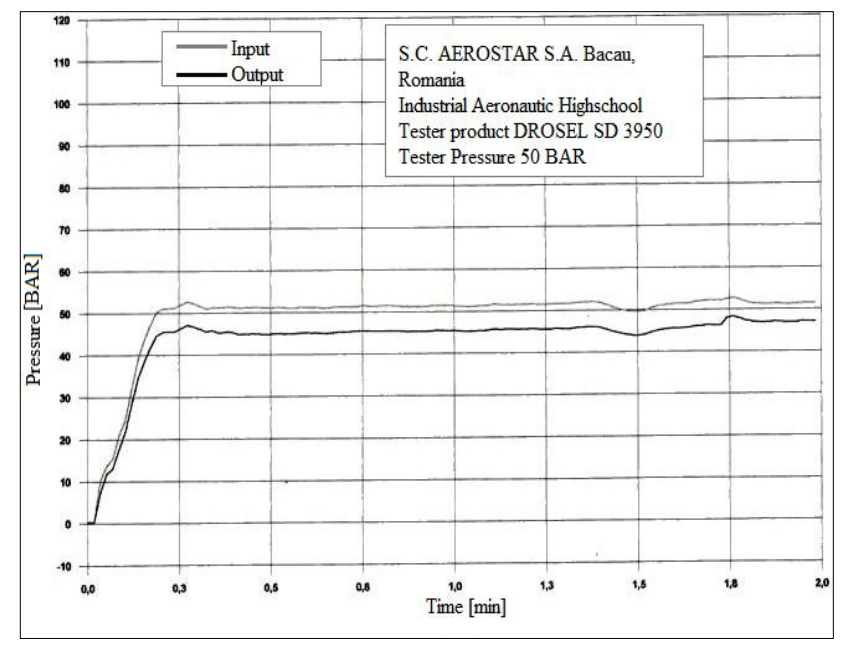

Fig. 6. Pressure drop on the throttle for $\mathrm{p}_{1}=50 \mathrm{bar}$

We got automatic diagrams for several values of the inlet pressure: 20, 50 (Fig. 6), 80 and 100 bars (Fig. 5), marking out the pressure fall on throttle level.

\section{CONCLUSIONS}

The experimental research emphasized the following conclusions concerning the efficiency of the diaphragms throttle valve use:

- $\quad$ in the case of low pressure values of about 20 bar, the adjustment is not significant; it becomes noticeable for 50 bar, when results a pressure fall around 8 bar;

- $\quad$ for an inlet pressure value of 80 bar results a pressure drop of 10 bars and for an inlet pressure value of 100 bar, the pressure fall on the throttle was of approximately 20 bar;

- to obtain a good motors speed control, the use of diaphragms throttle valve is recommended for pressure greater than $100 \mathrm{bar}$;

- finesse and precision of adjustment is important in high level installations.

Further research will diversify the range of values for the throttle flow and will compare the results achieved through the experimental research with the results of the simulations made in MATLAB.

\section{ACKNOWLEDGEMENTS}

The present work has been developed thanks to the Grant (CNCSIS) PNII, 2703/22-111/2008.

I want to thank to the people from Reliability Laboratory at S.C.Aerostar S.A. Bacau for the support given to achievement this study.

\section{REFERENCES}

Oroveanu, T. (1967). Mechanics of viscous fluids, Academy Pulishing House

Tita, I. (2009). Hydraulic and pneumatic transmissions, Publishing House PIM, ISBN 978-606-520-357-0, Iasi

Topliceanu , L.; Fita, M. (2003). Hydraulic drives, Publishing House Tehnica -Info, ISBN 9975-63-225-4, Chisinau

Vasiliu, N., Vasiliu, D. (1993). Computer Aided Design of Hydraulic Power Systems with Power Governor. Proceedings of the VIII-th International Conference on Pneumatics and Hydraulics, Miskolc

*** MathWorks, Available at http://www.mathworks.com/ 